\title{
Consommation d'alcool et lignes directrices pour la consommation d'alcool à faible risque chez les adultes : une analyse transversale de l'Alberta's Tomorrow Project
}

\author{
Darren R. Brenner*, Ph. D. (1,2); Tiffany R. Haig*, B.A. (3); Abbey E. Poirier, M. Sc. (1); Alianu Akawung, M. Sc. (3); \\ Christine M. Friedenreich, Ph. D. (1,2); Paula J. Robson, Ph. D., RNutr (R.-U.) (3,4)
}

Cet article a fait l'objet d'une évaluation par les pairs.

Diffuser cet article sur Twitter

\section{Résumé}

Introduction : La consommation modérée ou élevée d'alcool constitue un facteur de risque de mortalité toutes causes confondues et de cancer. Nous disposons de données transversales grâce aux enquêtes nationales, mais jusqu'à présent aucunes données sur la consommation d'alcool en Alberta n'étaient disponibles pour une grande cohorte prospective. Notre objectif est de définir, à la lumière des lignes directrices de prévention du cancer, les niveaux de consommation d'alcool des adultes ayant pris part à l'Alberta's Tomorrow Project. Nous avons également analysé les liens entre la consommation d'alcool et certains autres comportements à risque, en particulier à risque élevé.

Méthodologie : Entre 2001 et 2009, 31072 hommes et femmes de 35 à 69 ans ont participé à l'Alberta's Tomorrow Project, une grande étude de cohorte provinciale. Des données concernant la consommation d'alcool dans les 12 derniers mois ont été obtenues pour 26842 participants ayant répondu à des questionnaires autoadministrés sur la santé et sur le mode de vie. Nous avons effectué des analyses transversales sur la consommation d'alcool quotidienne et les lignes directrices de prévention du cancer en matière de consommation d'alcool en lien avec des facteurs sociodémographiques. Nous avons aussi examiné la prévalence combinée de la consommation d'alcool, du tabagisme, de l'obésité et de diverses comorbidités.

Résultats : Environ $14 \%$ des hommes et $12 \%$ des femmes ont déclaré consommer davantage d'alcool que ce que préconisent les recommandations en matière de prévention du cancer. Une consommation plus importante d'alcool a été rapportée chez les plus jeunes, les citadins, les personnes à revenu plus élevé et celles consommant davantage de viande rouge. En outre, le volume de consommation quotidienne d'alcool était positivement associé au tabagisme, tant chez les hommes que chez les femmes. De manière générale, les hommes étaient plus susceptibles que les femmes de correspondre aux profils comportementaux à risque modéré et élevé ainsi que de consommer quotidiennement de l'alcool.

Conclusion : Malgré les messages de santé publique à propos de l'impact négatif de la consommation d'alcool, une proportion considérable de participants de l'Alberta's Tomorrow Project consommait davantage d'alcool que ce que préconisent les recommandations en matière de prévention du cancer. Des stratégies durables en faveur d'une consommation d'alcool à faible risque auprès de ceux qui choisissent de boire pourraient avoir une influence sur le risque de maladie chronique au sein de cette population.

Mots-clés : alcool, cancer, Alberta's Tomorrow Project, cohorte, lignes directrices, prévention
Points saillants

- La consommation d'alcool constitue un facteur de risque de différentes maladies chroniques et de mortalité toutes causes confondues.

- Nous avons mesuré les niveaux de consommation d'alcool pour 31072 participants (de 2001 à 2009) de l'Alberta's Tomorrow Project, une cohorte d'adultes de 35 à 69 ans répartis géographiquement.

- Quatorze pour cent des hommes et $12 \%$ des femmes ont déclaré avoir consommé davantage d'alcool que ce que préconisent les recommandations en matière de prévention du cancer.

- Les niveaux élevés de consommation d'alcool ont été positivement associés au tabagisme et à d'autres facteurs de risque de maladie chronique.

- Les messages de santé publique devraient continuer à promouvoir une consommation d'alcool minime ou à faible risque pour réduire le fardeau des maladies chroniques en Alberta.

\section{Introduction}

L'alcool contribue de façon substantielle à diverses causes de mortalité : on estime qu'à l'échelle mondiale, l'alcool est lié à $25,8 \%$ des décès attribuables à une blessure, à 33,4\% des décès dus au diabète et aux maladies cardiovasculaires et à 12,5\%

Rattachement des auteurs :

1. Département d'épidémiologie du cancer et de recherche préventive, CancerControl Alberta, Alberta Health Services, Calgary (Alberta), Canada

2. Département d'oncologie et Département des sciences de la santé communautaire, École de médecine Cumming, Université de Calgary, Calgary (Alberta), Canada

3. Mesure, résultats, recherche et évaluation en matière de cancer (C-MORE), CancerControl Alberta, Alberta Health Services, Calgary (Alberta), Canada

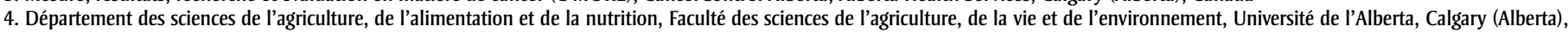
Canada

* Les deux premiers auteurs ont contribué également à cet article.

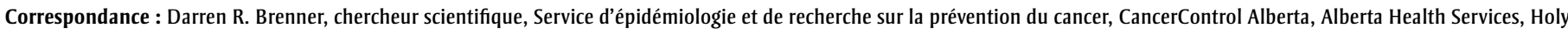
Cross Centre, bureau 514, boîte ACB 2210, 2nd Street SW, Calgary (Alberta) T2S 3C3; courriel : Darren.Brenner@ahs.ca 
des décès par cancer ${ }^{1}$. La consommation régulière d'alcool constitue un facteur de risque connu pour au moins huit types de cancer spécifiques, soit les cancers de la cavité buccale, de l'œsophage, du pharynx, du larynx, du sein chez la femme, de l'estomac et du foie et le cancer colorec$\mathrm{tal}^{2,3}$. Le Centre international de Recherche sur le Cancer (CIRC) a établi que l'éthanol (le métabolite actif de la consommation d'alcool) est un agent cancérogène pour l'être humain du groupe $1^{4}$, et qu'il existe suffisamment de preuves d'une relation dose-risque entre l'alcool et certains effets indésirables sur la santé, en particulier le cancer $^{5-9}$, sans signe d'effet de seuil ${ }^{2}$. En outre, il ne semble pas y avoir de différence appréciable selon le type de boisson $^{2}$. D'après les estimations récentes à l'échelle de la population du risque attribuable, 4,2 \% de tous les cas incidents de cancer dans la province de l'Alberta étaient attribuables à la consommation d'alcool en $2012^{10}$.

À l'inverse, il a été démontré que la consommation d'alcool faible ou modérée a un effet cardioprotecteur ${ }^{11-14}$ et fournit une protection contre le diabète de type $\mathrm{II}^{15,16}$ et contre d'autres maladies chroniques ${ }^{14,17}$. Cependant, diverses études récentes ont remis en question ces conclusions et indiquent qu'il n'y a aucune limite absolue de consommation, surtout pour le cancer $^{18-21}$. En dépit de cette controverse, définir un seuil sécuritaire fondé sur une méthodologie fiable, qui prenne en considération le type de boisson, la fréquence et le volume de consommation ainsi que les habitudes de consommation d'alcool, constitue une question de recherche importante $^{21}$. Des analyses récentes sur le sujet semblent indiquer qu'une consommation d'alcool même faible ou modérée n'offre aucune protection contre les maladies chroniques $^{21}$. Ces conclusions sont en contradiction avec les messages en vigueur en matière de directives de consommation d'alcool, qui encouragent les personnes qui choisissent de boire à consommer modérément de l'alcool ${ }^{3,22}$. Même si le pourcentage de Canadiens de 15 ans ou plus ayant consommé de l'alcool au cours de l'année précédente est passé de $79 \%$ en 2004 à $76 \%$ en 2013, les taux de comportements de consommation à risque ont augmenté23. Par exemple, les Directives de consommation d'alcool à faible risque du Canada recommandent aux femmes de ne pas consommer plus de 10 verres par semaine (avec un maximum de deux verres par jour) et aux hommes, de ne pas consommer plus de 15 verres par semaine (avec un maximum de 3 verres par jour) ${ }^{24,25}$. Malgré ces directives de consommation d'alcool à faible risque quant aux effets à long terme sur la santé (par exemple : cancer, épilepsie, pancréatite, insuffisance de poids à la naissance, accident vasculaire cérébral hémorragique, dysrythmies, cirrhose hépatique et hypertension), la proportion de Canadiens outrepassant ces directives de consommation d'alcool à faible risque continue d'augmenter : elle est passée, chez les 25 ans et plus qui consomment de l'alcool, de $13,0 \%$ en $2004^{26}$ à $17,6 \%$ en $2012^{27}$ et à $20,0 \%$ en $2013^{28}$.

Les estimations antérieures de la prévalence de la consommation d'alcool en Alberta sont issues d'enquêtes nationales sur l'usage de drogue et d'alcool ${ }^{26,28-34}$. Des données transversales sur la consommation d'alcool en Alberta étaient disponibles grâce à ces enquêtes nationales, mais aucunes données n'étaient disponibles pour une grande cohorte prospective. L'objectif de nos analyses était de définir, à la lumière des lignes directrices sur la prévention $\mathrm{du}$ cancer, les niveaux de consommation d'alcool chez les adultes ayant pris part à l'Alberta's Tomorrow Project. En outre, nous avons cerné les facteurs sociodémographiques associés aux habitudes de consommation d'alcool, nous avons noté la prévalence de la consommation d'alcool combinée au tabagisme et aux profils à risque élevé et nous avons évalué la proportion de participants qui outrepassaient les recommandations en matière de consommation d'alcool du World Cancer Research Fund et de l'American Institute of Cancer Research (WCRF/AICR).

\section{Méthodologie}

L'Alberta's Tomorrow Project est une étude de cohorte prospective longitudinale créée en vue d'examiner les liens entre différents facteurs liés au mode de vie et les maladies chroniques et qui comprend actuellement 55000 Albertains de 35 à 69 ans. De l'information détaillée sur les méthodes de recrutement de l'Alberta's Tomorrow Project a déjà été publiée ${ }^{35,36}$. En bref, les participants de l'Alberta's Tomorrow Project ont été recrutés par composition téléphonique aléatoire entre 2001 et 2009. Lors de ce processus, 63486 personnes ont manifesté leur intérêt à participer et 48,8 \% d'entre elles se sont inscrites dans la cohorte, soit 31072 participants $^{36}$. Les participants ont répondu à des questionnaires autoadministrés, notamment le Health and Lifestyle Questionnaire, le Diet History Questionnaire ${ }^{37}$, et le Past Year Total Physical Activity Questionnaire ${ }^{38,39}$. Ces questionnaires ont permis de recueillir de l'information sur les antécédents médicaux personnels et familiaux des participants, leurs comportements en matière de dépistage du cancer, leurs habitudes alimentaires, leur consommation d'alcool et de tabac ainsi que les expositions environnementales. Nos analyses n'incluent que les participants recrutés au cours de la première phase de l'étude et ayant répondu au Health and Lifestyle Questionnaire et au Diet History Questionnaire. Des 31072 participants de la cohorte, $86 \%(n=26842)$ ont fourni de l'information sur leur consommation d'alcool.

\section{Évaluation de la consommation d'alcool et des variables d'intérêt}

L'information sur la consommation d'alcool a été recueillie de 2001 à 2009 à l'aide d'un questionnaire de fréquence alimentaire (QFA) axé sur les aptitudes cognitives et préparé par le United States National Cancer Institute. Cet outil d'évaluation nutritionnelle porte sur les 12 mois précédents $^{40}$ et a été adapté pour le Canada ${ }^{37}$. Le Diet History Questionnaire (DHQ) a été analysé à l'aide du logiciel Diet*Calc, version 1.4.2 (version canadienne). Il a été validé pour tous les nutriments et groupes alimentaires, dont l'alcool. De plus, de nombreuses autres études de bonne qualité ont utilisé des QFA dans leur évaluation de la consommation d'alcool ${ }^{12,41,42}$. Les participants ont été interrogés sur la fréquence et le volume de leur consommation de bière, de vin (et de cooler au vin) et de spiritueux (et cocktails) au cours de l'année précédente. Le questionnaire comprenait des questions sur les cannettes ou bouteilles de bière (12 onces), les verres de vin ou cooler au vin ( 5 onces) et les spiritueux ou cocktails $(1,5$ once). Pour chaque type de boisson, 10 catégories de réponse étaient possibles quant à la fréquence, allant de jamais à six portions (verres) ou plus par jour au cours de l'année précédente. Nous avons estimé la quantité moyenne d'éthanol consommé par semaine en utilisant la norme canadienne de 13,6 g d'éthanol pour une consommation standard, ce qui correspond à environ $341 \mathrm{ml}$ de bière, $142 \mathrm{ml}$ de vin et $43 \mathrm{ml}$ de spiritueux ${ }^{43}$. Il a été impossible de recueillir de l'information sur la consommation excessive épisodique ou de savoir si les participants consommaient surtout les jours de semaine ou la 
fin de semaine. Nous avons estimé la proportion de participants respectant les recommandations du WCRF/AICR en matière de prévention du cancer et la proportion de ceux ayant consommé au-delà de ces recommandations ${ }^{44}$. Ils ont été répartis en deux catégories : ceux ayant respecté les recommandations (2 consommations par jour ou moins pour les hommes; 1 consommation par jour ou moins pour les femmes) et ceux les ayant outrepassées (plus de 2 consommations par jour pour les hommes et plus d'1 consommation par jour pour les femmes).

Pour estimer la relation entre les habitudes de consommation d'alcool et le tabagisme, nous avons examiné la proportion d'hommes et de femmes respectant et outrepassant les lignes directrices de consommation d'alcool au sein des groupes de participants fumeurs. Les données relatives au tabagisme se fondent sur les réponses aux questionnaires autodéclarés remplis au début de l'étude, les participants ayant indiqué quelles étaient leurs habitudes actuelles et passées en matière de tabagisme. Ils ont été répartis en 4 catégories : n'a jamais fumé, ex-fumeur, fumeur occasionnel et fumeur quotidien. L'indice de masse corporelle (IMC) a été déterminé à partir des mesures de la taille et du poids fournis par les participants et les données concernant la comorbidité sont fondées sur le diagnostic d'un médecin rapporté par les participants dans le questionnaire de départ. Afin d'évaluer la prévalence des facteurs de risque multiples, nous avons pris en considération le tabagisme, le poids (surpoids ou obésité, soit un indice de masse corporelle [IMC] supérieur à $25 \mathrm{~kg} / \mathrm{m}^{2}$ ) et la comorbidité (autodéclaration d'une maladie chronique, soit l'hypertension artérielle, l'angor, un taux de cholestérol élevé, une crise cardiaque, un accident vasculaire cérébral, le diabète, des polypes du côlon, la rectocolite hémorragique et la cirrhose hépatique). Les facteurs de risque multiples ont été répartis en quatre catégories : aucun risque (les participants ne répondaient à aucun des critères, c'est-à-dire qu'ils étaient nonfumeurs, avaient un IMC inférieur à $25 \mathrm{~kg} / \mathrm{m}^{2}$ et ne présentaient aucune affection chronique), risque faible (un des trois critères), risque modéré (deux des trois critères) et risque élevé (les trois critères). Nous avons ensuite examiné, à l'intérieur de ces catégories de niveau de risque, la proportion d'hommes et de femmes qui respectaient et outrepassaient les lignes directrices sur la consommation d'alcool à faible risque.

\section{Analyses statistiques}

Nous avons utilisé des statistiques descriptives pour caractériser les habitudes de consommation au sein de la cohorte et nous avons examiné la consommation quotidienne moyenne d'alcool $(0 ; 0,1$ à 4,$9 ; 5$ à 14,$9 ; 15$ à 29,9; 30 à 44,$9 ; 45$ g et plus). Nous avons calculé les moyennes et les écarts-types pour les variables continues ainsi que les fréquences et les pourcentages pour les variables nominales. Un test de concordance kappa a également été effectué pour établir la concordance entre l'estimation de la quantité d'alcool en fonction du nombre de verres par jour fournie par Diet*Calc et le nombre de grammes d'éthanol par jour (1 verre = 13,6 g d'éthanol). Nous avons utilisé des tests du khi carré de Pearson pour toutes les analyses comparatives. Nous avons eu de plus recours à des modèles de régression logistique à variables multiples pour établir des associations entre les caractéristiques sociodémographiques et les recommandations de consommation d'alcool établies par le WCRF. Les données manquantes représentaient moins de $1 \%$ pour toutes les variables comprises dans l'étude. Les valeurs manquantes ont été exclues des analyses. Tous les tests statistiques ont été effectués avec un seuil de signification de $5 \%$ à l'aide du système d'analyse SAS version 9.2 (SAS Institute, Cary, Caroline du Nord, États-Unis) avec un système d'exploitation Linux.

\section{Résultats}

\section{Habitudes de consommation d'alcool}

La majorité des participants $(84 \%, \mathrm{n}=$ 22 627) ont indiqué avoir consommé de l'alcool au cours des 12 derniers mois. Le tableau 1 présente la proportion de participants à l'étude Alberta's Tomorrow Project pour chaque catégorie de consommation d'alcool en fonction de leur sexe et de leurs caractéristiques sociodémographiques. La consommation d'alcool médiane (écart interquartile) atteignait 2,1 $(5,8) \mathrm{g}$ par jour pour les femmes et $5,9(14,8) \mathrm{g}$ par jour pour les hommes. Les hommes et les femmes qui consommaient de l'alcool avaient davantage tendance à être plus jeunes, à consommer davantage de viande rouge, à être d'origine ethnique européenne, à vivre en milieu urbain, à travailler à temps plein et à avoir un revenu familial annuel de plus de $80000 \$$. Nous avons observé une association positive marquée entre la consommation quotidienne d'alcool et le tabagisme, autant chez les hommes que chez les femmes.

\section{Recommandations en matière de consommation d'alcool du World Cancer Research Fund pour la prévention du cancer}

Le tableau 2 présente la proportion d'hommes et de femmes qui respectaient ou outrepassaient les recommandations du World Cancer Research Fund en matière la consommation personnelle d'alcool, par catégorie démographique et en fonction de la consommation d'alcool autodéclarée. La majorité (87 \%) des participants de la cohorte ayant déclaré avoir consommé de l'alcool dans les 12 derniers mois respectaient les recommandations personnelles liées à la consommation d'alcool alors que $13 \%$ les outrepassaient. Parmi ces derniers, il y avait légèrement moins de femmes $(12,1 \%)$ que d'hommes $(13,6 \%)$. On a pu observer une plus grande proportion d'hommes consommant de l'alcool au-delà des recommandations chez ceux qui étaient plus éduqués, avaient des revenus familiaux annuels plus élevés, étaient d'âge moyen (entre 45 et 54 ans) et étaient divorcés, séparés ou veufs. Les femmes qui outrepassaient les lignes directrices de consommation d'alcool avaient, de la même manière, des revenus familiaux plus élevés, étaient employées à temps plein ou retraitées, et étaient âgées de 45 à 54 ans.

Les associations entre les lignes directrices en matière de consommation d'alcool du WCRF et les caractéristiques sociodémographiques sont présentées dans le tableau 3. Dans l'ensemble, les hommes et les femmes ayant un revenu familial plus élevé avaient plus de chances d'outrepasser les lignes directrices de consommation d'alcool du WCRF. De plus, les participants qui avaient déjà fumé (fumeurs quotidiens, fumeurs occasionnels et ex-fumeurs) avaient plus de chances que ceux qui n'avaient jamais fumé d'outrepasser les lignes directrices en matière de consommation d'alcool du WCRF $(p<0,0001)$. Chez les hommes, ces proportions étaient plus élevées chez les fumeurs quotidiens (rapport de cotes $[\mathrm{RC}]=3,61$, IC à $95 \%: 3,00$ à 4,36) et occasionnels $(\mathrm{RC}=3,56$, IC à $95 \%: 2,63$ à 4,82). Chez les femmes, des résultats semblables ont été observés chez celles qui fumaient quotidiennement $(\mathrm{RC}=3,06$, IC 
TABLEAU 1

Caractéristiques des participants selon les habitudes de consommation d'alcool déclarées (g/jour)

\begin{tabular}{|c|c|c|c|c|c|c|c|}
\hline \multirow{2}{*}{ Caractéristiques } & \multicolumn{6}{|c|}{ Consommation quotidienne totale d'alcool (g/jour) } & \multirow{2}{*}{ Valeur $p^{c}$} \\
\hline & 0 & 0,1 à 4,9 & 5 à 14,9 & 15 à 29,9 & 30 à 44,9 & $\geq 45$ & \\
\hline Hommes & $n=1342$ & $\mathrm{n}=3327$ & $\mathrm{n}=2708$ & $n=1546$ & $\mathrm{n}=433$ & $\mathrm{n}=758$ & \\
\hline Origine ethnique européenne (\%) & 69,5 & 73,8 & 76,8 & 78,1 & 80,1 & 76,1 & $<0,0001$ \\
\hline Antécédents familiaux de cancer (\%) & 51,3 & 48,6 & 51,0 & 53,0 & 54,3 & 52,6 & 0,0247 \\
\hline $\begin{array}{l}\text { Antécédents de coloscopie ou de } \\
\text { sigmoïdoscopie (\%) }\end{array}$ & 21,3 & 20,7 & 20,4 & 22,1 & 22,9 & 19,8 & 0,158 \\
\hline Fumeur quotidien (\%) & 12,5 & 12,4 & 12,5 & 15,7 & 18,7 & 31,1 & $<0,0001$ \\
\hline Diplôme d'études postsecondaires (\%) & 52,6 & 55,2 & 59,6 & 59,8 & 56,4 & 52,0 & $<0,0001$ \\
\hline Revenu familial $\geq 80000$ \$ (\%) & 24,7 & 33,3 & 42,5 & 47,0 & 45,3 & 36,4 & $<0,0001$ \\
\hline Emploi à temps plein (\%) & 68,6 & 74,3 & 79,2 & 75,3 & 73,9 & 76,8 & $<0,0001$ \\
\hline Marié/conjoint de fait (\%) & 82,3 & 83,3 & 85,2 & 83,2 & 79,0 & 80,7 & 0,0024 \\
\hline Vit en milieu urbain (\%) & 70,8 & 77,3 & 78,6 & 81,2 & 79,2 & 76,7 & $<0,0001$ \\
\hline Âge (ans) & $52,1(9,4)$ & $50,6(9,4)$ & $49,7(8,8)$ & $50,5(8,9)$ & $50,5(8,9)$ & $49,9(8,7)$ & $<0,0001$ \\
\hline Indice de masse corporelle & $28,4(4,8)$ & $28,3(4,7)$ & $28,0(4,2)$ & $27,7(4,0)$ & $28,2(4,0)$ & $27,8(4,0)$ & $<0,0001$ \\
\hline $\begin{array}{l}\text { Activités physiques récréatives } \\
\text { (MET h/semaine) }\end{array}$ & $22,4(24,7)$ & $25,0(26,3)$ & $30,9(27,7)$ & $31,9(27,4)$ & $32,3(30,6)$ & $26,8(27,9)$ & $<0,0001$ \\
\hline $\begin{array}{l}\mathrm{N}^{\text {bre }} \text { de paquets-années parmi les } \\
\text { fumeurs ou ex-fumeurs }\end{array}$ & $34,5(10,3)$ & $32,2(10,4)$ & $29,5(9,6)$ & $29,2(9,5)$ & $29,7(9,1)$ & $30,7(9,0)$ & $<0,0001$ \\
\hline $\begin{array}{l}\text { Apport calorique de source autre } \\
\text { que l'alcool (kcal/jour) }\end{array}$ & $2185,1(1110,6)$ & $2046,7(878,9)$ & $2076,6(850,5)$ & $2084,6(820,2)$ & $2250,8(951,3)$ & $2495,7(1059,1)$ & $<0,0001$ \\
\hline $\begin{array}{l}\text { Viande rouge dans le régime } \\
\text { alimentaire ( } n^{\text {bre }} \text { portions/semaine) }\end{array}$ & $5,7(5,2)$ & $5,4(4,2)$ & $5,8(4,1)$ & $6,1(4,1)$ & $6,8(5,2)$ & $7,7(5,5)$ & $<0,0001$ \\
\hline $\begin{array}{l}\text { Indice de saine alimentation - } \\
\text { Canada, } 2005^{\mathrm{b}}\end{array}$ & $51,1(9,6)$ & $50,8(9,3)$ & $50,9(8,7)$ & $50,5(8,3)$ & $50,6(8,0)$ & $50,3(7,5)$ & 0,272 \\
\hline Femmes & $n=2873$ & $n=8688$ & $\mathrm{n}=3346$ & $n=1329$ & $\mathrm{n}=201$ & $\mathrm{n}=291$ & \\
\hline Origine ethnique européenne (\%) & 72,9 & 77,8 & 80,2 & 80,4 & 83,1 & 85,2 & $<0,0001$ \\
\hline Antécédents familiaux de cancer (\%) & 55,5 & 55,2 & 52,9 & 54,7 & 54,7 & 51,2 & 0,2011 \\
\hline $\begin{array}{l}\text { Antécédents de coloscopie ou de } \\
\text { sigmoïdoscopie (\%) }\end{array}$ & 28,1 & 24,4 & 23,3 & 25,2 & 20,9 & 22,3 & 0,0002 \\
\hline Fumeuse quotidienne (\%) & 13,0 & 13,9 & 13,3 & 17,5 & 22,9 & 36,8 & $<0,0001$ \\
\hline Diplôme d'études postsecondaires (\%) & 41,6 & 47,7 & 55,0 & 52,0 & 44,8 & 38,1 & $<0,0001$ \\
\hline Revenu familial $\geq 80000 \$(\%)$ & 16,9 & 27,9 & 38,9 & 39,1 & 34,8 & 34,0 & $<0,0001$ \\
\hline Emploi à temps plein (\%) & 34,1 & 45,4 & 47,5 & 46,0 & 52,7 & 50,5 & $<0,0001$ \\
\hline Mariée/conjointe de fait (\%) & 74,2 & 74,9 & 79,0 & 79,6 & 77,6 & 74,2 & $<0,0001$ \\
\hline Vit en milieu urbain (\%) & 67,1 & 76,2 & 80,3 & 81,9 & 75,6 & 77,7 & $<0,0001$ \\
\hline Âge (ans) & $51,9(9,5)$ & $50,2(9,3)$ & $49,2(8,7)$ & $50,7(9,0)$ & $48,6(8,4)$ & $49,8(8,2)$ & $<0,0001$ \\
\hline Indice de masse corporelle & $28,5(6,9)$ & $27,6(6,1)$ & $26,2(5,0)$ & $25,8(4,7)$ & $25,9(4,6)$ & $26,8(5,1)$ & $<0,0001$ \\
\hline $\begin{array}{l}\text { Activités physiques récréatives } \\
\text { (MET h/semaine) }\end{array}$ & $17,9(20,6)$ & $22,5(22,9)$ & $27,3(24,2)$ & $29,2(25,2)$ & $28,4(24,6)$ & $20,9(22,7)$ & $<0,0001$ \\
\hline $\begin{array}{l}\mathrm{N}^{\text {bre }} \text { de paquets-années parmi les } \\
\text { fumeuses ou ex-fumeuses }\end{array}$ & $32,3(10,1)$ & $30,3(9,7)$ & $28,5(9,1)$ & $29,6(9,5)$ & $28,9(7,4)$ & $30,8(8,5)$ & $<0,0001$ \\
\hline $\begin{array}{l}\text { Apport calorique de source autre } \\
\text { que l'alcool (kcal/jour) }^{\mathrm{a}}\end{array}$ & $1644,2(720,8)$ & $1574,9(634,0)$ & $1579,4(604,3)$ & $1613,4(629,7)$ & $1681,2(584,4)$ & $1782,8(793,4)$ & $<0,0001$ \\
\hline $\begin{array}{l}\text { Viande rouǵe dans le régime } \\
\text { alimentaire ( } n^{\text {bre }} \text { portions/semaine) }\end{array}$ & $3,3(2,7)$ & $3,4(2,5)$ & $3,6(2,5)$ & $3,7(2,5)$ & $4,6(3,0)$ & $4,0(2,6)$ & $<0,0001$ \\
\hline $\begin{array}{l}\text { Indice de saine alimentation - } \\
\text { Canada, } 2005^{\text {b }}\end{array}$ & $55,3(10,1)$ & $55,4(9,7)$ & $55,3(8,9)$ & $54,4(8,5)$ & $53,6(7,4)$ & $52,8(7,3)$ & $<0,0001$ \\
\hline
\end{tabular}

Suite à la page suivante 
TABLEAU 1 (suite)

Caractéristiques des participants selon les habitudes de consommation d'alcool déclarées (g/jour)

\begin{tabular}{|c|c|c|c|c|c|c|c|}
\hline \multirow{2}{*}{ Caractéristiques } & \multicolumn{6}{|c|}{ Consommation quotidienne totale d'alcool (g/jour) } & \multirow{2}{*}{ Valeur $\boldsymbol{p}^{\mathrm{c}}$} \\
\hline & 0 & 0,1 à 4,9 & 5 à 14,9 & 15 à 29,9 & 30 à 44,9 & $\geq 45$ & \\
\hline Postménopause (\%) & 45,4 & 53,7 & 58,1 & 51,3 & 62,2 & 50,5 & $<0,0001$ \\
\hline $\begin{array}{l}\text { Utilisation actuelle } \\
\text { d'hormonothérapie (\%) }\end{array}$ & 16,3 & 14,8 & 13,8 & 17,0 & 10,5 & 15,1 & 0,0285 \\
\hline $\begin{array}{l}\text { Mammographie dans les } 3 \text { dernières } \\
\text { années (\%) }\end{array}$ & 79,9 & 81,5 & 82,3 & 85,7 & 81,4 & 74,9 & $<0,0001$ \\
\hline
\end{tabular}

Abréviation : MET h/semaine, équivalent métabolique de la tâche en heures par semaine.

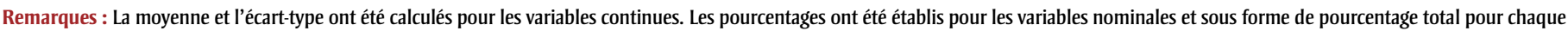
colonne, soit $100 \%$ à l'intérieur de chaque catégorie de consommation d'alcool.

a $1 \mathrm{kcal}=4,18 \mathrm{~kJ}$.

${ }^{\mathrm{b}}$ Sans consommation d'alcool.

' Le test du khi carré a été utilisé pour les variables nominales, et l'analyse de variance à un facteur a été utilisée pour les variables continues.

à $95 \%: 2,62$ à 3,59) et occasionnellement $(\mathrm{RC}=3,20$, IC à $95 \%: 2,43$ à 4,21), et les femmes non européennes ou d'origine ethnique mixte avaient moins tendance que celles d'origine européenne à outrepasser les lignes directrices $(\mathrm{RC}=0,66, \mathrm{IC}$ à $95 \%$ : $0,51$ à 0,85$)$.

\section{Habitudes de consommation d'alcool et autres comportements à risque}

Comme l'illustre le tableau 4, une plus grande proportion de non-fumeurs a été observée parmi ceux qui ne consommaient pas d'alcool. On a pu observer une association positive entre le fait de fumer et la consommation quotidienne totale d'alcool.
Le volume d'alcool consommé s'est révélé associé à des catégories de facteurs de risques multiples aussi bien chez les hommes que chez les femmes.

Presque 31,0 \% des hommes et 25,4\% des femmes qui outrepassaient les lignes directrices de consommation d'alcool fumaient (tableau 5). L'analyse des facteurs de risque multiples a révélé qu'une plus grande proportion d'hommes (56,0 \%) que de femmes $(34,6 \%)$ outrepassaient les lignes directrices de consommation d'alcool et présentaient des profils de risque modéré ou élevé. La prévalence des facteurs de risque multiples était légèrement plus faible chez les femmes qui outrepassaient les recommandations (35\%) que chez celles qui les respectaient (37\%).

\section{Analyse}

Nous avons observé que la majorité des participants de la cohorte (84\%) avaient consommé de l'alcool dans les 12 derniers mois, ce qui est légèrement plus élevé que ce qui a été rapporté dans d'autres études sur la consommation d'alcool en Alberta $(76 \%)^{45}$ et au Canada $(77,1 \%)^{46}$. La plupart des participants qui ont indiqué avoir consommé de l'alcool dans les 12 derniers mois n'ont pas outrepassé les recommandations en matière de consommation d'alcool à faible risque établies par

TABLEAU 2

Proportion des participants à l'étude Alberta's Tomorrow Project qui respectaient ou qui outrepassaient les recommandations de consommation d'alcool du World Cancer Research Fund/American Institute for Cancer Research selon les caractéristiques sociodémographiques

\begin{tabular}{|c|c|c|c|c|c|c|}
\hline & \multicolumn{3}{|c|}{ Hommes $(n=10114)$} & \multicolumn{3}{|c|}{ Femmes $(n=16728)$} \\
\hline & $\begin{array}{c}\text { Respecte les } \\
\text { lignes directrices } \\
\end{array}$ & $\begin{array}{c}\text { Dépasse les lignes } \\
\text { directrices }^{c}\end{array}$ & \multirow[t]{2}{*}{ Valeur $\boldsymbol{p}^{\mathrm{d}}$} & $\begin{array}{c}\text { Respecte les } \\
\text { lignes directrices }\end{array}$ & $\begin{array}{c}\text { Dépasse les lignes } \\
\text { directrices }^{c}\end{array}$ & \multirow[t]{2}{*}{ Valeur $\boldsymbol{p}^{\mathrm{d}}$} \\
\hline & n (\%) & n (\%) & & n (\%) & n (\%) & \\
\hline Totaux & $8744(86,5)$ & $1370(13,6)$ & & $14708(87,9)$ & $2020(12,1)$ & \\
\hline \multicolumn{7}{|l|}{ Âge (ans) } \\
\hline 35 à 44 & $2648(30,3)$ & $410(29,9)$ & \multirow{4}{*}{0,0021} & $4680(31,8)$ & $597(29,6)$ & \multirow{4}{*}{$<0,0001$} \\
\hline 55 à 64 & $2231(25,5)$ & $324(23,7)$ & & $3650(24,8)$ & $459(22,7)$ & \\
\hline 65 à 69 & $792(9,1)$ & $94(6,9)$ & & $1320(9,0)$ & $155(7,7)$ & \\
\hline Données manquantes & $0(0,0)$ & $0(0,0)$ & & $0(0,0)$ & $0(0,0)$ & \\
\hline \multicolumn{7}{|l|}{ Études ${ }^{\mathrm{e}}$} \\
\hline Aucun diplôme d'études secondaires & $914(10,5)$ & $144(10,5)$ & & $1359(9,2)$ & $151(7,5)$ & \multirow{2}{*}{0,0731} \\
\hline Données manquantes & $1(0,01)$ & $0(0,0)$ & & $1(0,01)$ & $0(0,0)$ & \\
\hline
\end{tabular}

Suite à la page suivante 
TABLEAU 2 (suite)

Proportion des participants à l'étude Alberta's Tomorrow Project qui respectaient ou qui outrepassaient les recommandations de consommation d'alcool du World Cancer Research Fund/American Institute for Cancer Research selon les caractéristiques sociodémographiques ${ }^{\mathrm{a}}$

\begin{tabular}{|c|c|c|c|c|c|c|}
\hline & \multicolumn{3}{|c|}{ Hommes $(n=10114)$} & \multicolumn{3}{|c|}{ Femmes $(n=16728)$} \\
\hline & $\begin{array}{c}\text { Respecte les } \\
\text { lignes directrices }^{b}\end{array}$ & $\begin{array}{c}\text { Dépasse les lignes } \\
\text { directrices }^{c}\end{array}$ & \multirow[t]{2}{*}{ Valeur $p^{d}$} & $\begin{array}{c}\text { Respecte les } \\
\text { lignes directrices }\end{array}$ & $\begin{array}{c}\text { Dépasse les lignes } \\
\text { directrices }^{c}\end{array}$ & \multirow[t]{2}{*}{ Valeur $p$} \\
\hline & n (\%) & n (\%) & & n (\%) & n (\%) & \\
\hline \multicolumn{7}{|l|}{ Revenu familialf $^{f}$} \\
\hline$<30000 \$$ & $804(9,2)$ & $88(6,4)$ & \multirow{4}{*}{0,0001} & $2373(16,1)$ & $220(10,9)$ & \multirow{4}{*}{$<0,0001$} \\
\hline $30000 \$$ à $49000 \$$ & $2189(25,0)$ & $298(21,8)$ & & $4265(29,0)$ & $499(24,7)$ & \\
\hline$\geq 80000 \$$ & $3224(36,9)$ & $564(41,2)$ & & $4131(28,1)$ & $764(37,8)$ & \\
\hline Données manquantes & $134(1,5)$ & $16(1,2)$ & & $443(3,0)$ & $50(2,5)$ & \\
\hline \multicolumn{7}{|l|}{ Situation professionnelle } \\
\hline Temps plein & $6563(75,1)$ & $1041(76,0)$ & \multirow{3}{*}{0,5928} & $6413(43,6)$ & $965(47,8)$ & \multirow{3}{*}{0,0005} \\
\hline Temps partiel & $563(6,4)$ & $93(6,8)$ & & $3419(23,3)$ & $437(21,6)$ & \\
\hline Données manquantes & $4(0,1)$ & $0(0,0)$ & & $8(0,1)$ & $0(0,0)$ & \\
\hline \multicolumn{7}{|l|}{ État matrimonial } \\
\hline Marié/conjoint de fait & $7324(83,8)$ & $1098(80,2)$ & \multirow{4}{*}{0,0011} & $11125(75,6)$ & $1589(78,7)$ & \multirow{4}{*}{0,0043} \\
\hline Célibataire (jamais marié) & $562(6,4)$ & $90(6,6)$ & & $817(5,6)$ & $79(3,9)$ & \\
\hline Divorcé/séparé/veuf & $857(9,8)$ & $182(13,3)$ & & $2764(18,8)$ & $352(17,4)$ & \\
\hline Données manquantes & $1(0,01)$ & $0(0,0)$ & & $2(0,01)$ & $0(0,0)$ & \\
\hline \multicolumn{7}{|l|}{ Catégorie de fumeur } \\
\hline Quotidien & $1135(13,0)$ & $342(25,0)$ & \multirow{2}{*}{$<0,0001$} & $1996(13,6)$ & $414(20,5)$ & \multirow{2}{*}{$<0,0001$} \\
\hline Occasionnel & $269(3,1)$ & $82(6,0)$ & & $379(2,6)$ & $98(4,9)$ & \\
\hline \multicolumn{7}{|l|}{ Zone géographique ${ }^{g}$} \\
\hline Milieu rural & $1975(22,6)$ & $307(22,4)$ & \multirow{3}{*}{0,8834} & $3628(24,7)$ & $399(19,8)$ & \multirow{3}{*}{$<0,0001$} \\
\hline Milieu urbain & $6769(77,4)$ & $1063(77,6)$ & & $11080(75,3)$ & $1621(80,3)$ & \\
\hline Données manquantes & $0(0,0)$ & $0(0,0)$ & & $0(0,0)$ & $0(0,0)$ & \\
\hline
\end{tabular}

Remarque : Les pourcentages totaux de chaque colonne ont été indiqués, soit $100 \%$ à l'intérieur de chaque catégorie relative aux lignes directrices de consommation d'alcool.

${ }^{a}$ Données présentées sous forme de nombres et de pourcentages.

${ }^{\mathrm{b}}$ Le respect des lignes directrices correspond à $\leq 2$ verres par jour chez les hommes et $\leq 1$ verre par jour chez les femmes.

' Le dépassement des lignes directrices correspond à $>2$ verres par jour chez les hommes et $>1$ verre par jour chez les femmes.

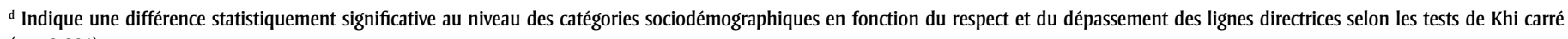
$(p<0,001)$.

${ }^{\text {e }}$ Réponses combinées : études collégiales ou de formation professionnelle non terminées, diplôme d'études collégiales ou de formation professionnelle, études universitaires terminées.

${ }_{\mathrm{f}}^{\mathrm{f}}$ Les données concernant le revenu proviennent de la réponse à une question sur le revenu familial total avant impôt, notamment.

\& L'emplacement géographique a été déterminé à l'aide des codes postaux, dans lesquels le « 0 » placé comme valeur numérique centrale indique qu'il s'agit d'une résidence rurale. 
TABLEAU 3

Associations entre les lignes directrices de consommation d'alcool du WCRF et les caractéristiques sociodémographiques des participants de l'étude de cohorte Alberta's Tomorrow Project

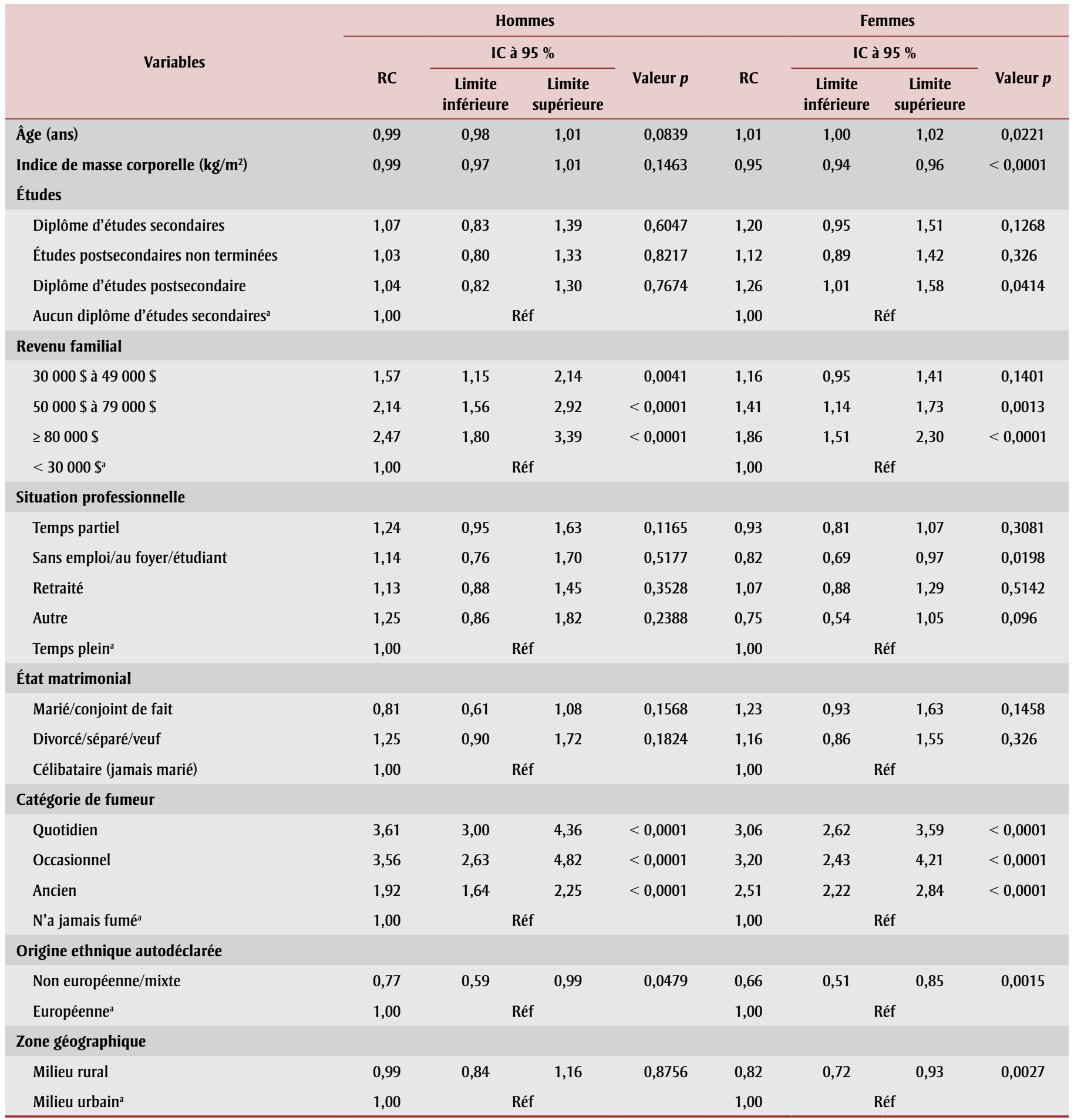

Abréviations : IC, intervalle de confiance; RC, rapport de cotes; Réf, catégorie de référence; WCRF, World Cancer Research Fund.

${ }^{a}$ Catégorie de référence. 
TABLEAU 4

Habitudes de consommation d'alcool autodéclarées et caractéristiques à risque qui y sont reliées dans la cohorte de l'Alberta's Tomorrow Project ${ }^{a}$

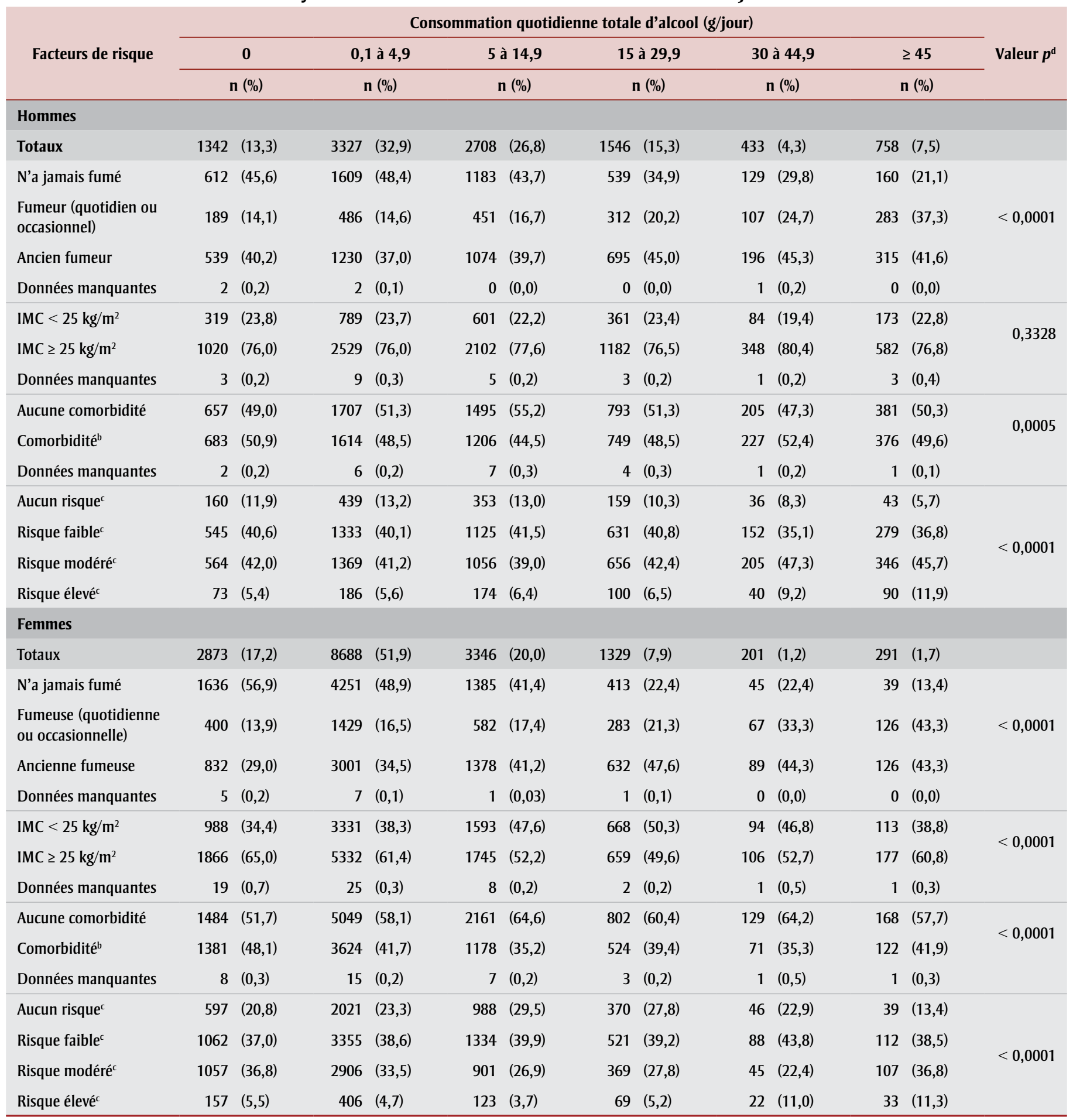

Abréviation : IMC, indice de masse corporelle.

Remarque : Les résultats sont présentés sous forme de pourcentage total pour chaque colonne, soit $100 \%$ à l'intérieur de chaque catégorie de consommation d'alcool.

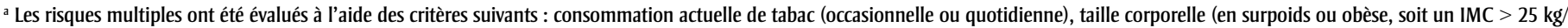
$\mathrm{m}^{2}$ ) et comorbidité.

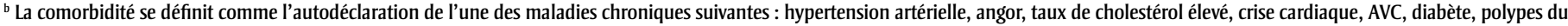
côlon, rectocolite hémorragique et cirrhose hépatique.

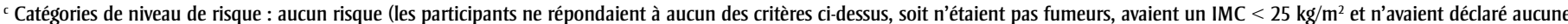

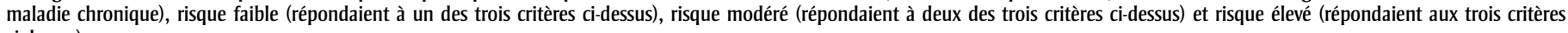
ci-dessus).

${ }^{\text {d }}$ Le test de Khi carré a été utilisé pour les variables nominales, et l'analyse de variance à un facteur a été utilisée pour les variables continues. 


\section{TABLEAU 5 \\ Respect des lignes directrices de consommation d'alcool du WCRF et caractéristiques à risque ${ }^{a}$ dans la cohorte de l'Alberta's Tomorrow Project}

\begin{tabular}{|c|c|c|c|c|c|}
\hline \multirow{3}{*}{ Facteurs de risque } & \multicolumn{4}{|c|}{$\begin{array}{c}\text { Lignes directrices de consommation } \\
\text { d'alcool du WCRF }\end{array}$} & \multirow{3}{*}{ Valeur $p$} \\
\hline & \multicolumn{2}{|c|}{$\begin{array}{c}\text { Respecte les lignes } \\
\text { directrices }\end{array}$} & \multicolumn{2}{|c|}{$\begin{array}{c}\text { Dépasse les lignes } \\
\text { directrices }\end{array}$} & \\
\hline & \multicolumn{2}{|c|}{$\mathrm{n}(\%)$} & \multicolumn{2}{|c|}{$\mathrm{n}(\%)$} & \\
\hline \multicolumn{6}{|l|}{ Hommes } \\
\hline Totaux & 8744 & $(86,5)$ & 1370 & $(13,6)$ & \multirow{5}{*}{$<0,0001$} \\
\hline N’a jamais fumé & 3882 & $(44,4)$ & 350 & $(25,6)$ & \\
\hline Fumeur (quotidien ou occasionnel) & 1404 & $(16,1)$ & 424 & $(31,0)$ & \\
\hline Ancien fumeur & 3454 & $(39,5)$ & 595 & $(43,4)$ & \\
\hline Données manquantes & 4 & $(0,1)$ & 1 & $(0,1)$ & \\
\hline $\mathrm{IMC}<25 \mathrm{~kg} / \mathrm{m}^{2}$ & 2025 & $(23,2)$ & 302 & $(22,0)$ & \multirow{3}{*}{0,3680} \\
\hline $\mathrm{IMC} \geq 25 \mathrm{~kg} / \mathrm{m}^{2}$ & 6699 & $(76,6)$ & 1064 & $(77,7)$ & \\
\hline Données manquantes & 20 & $(0,2)$ & 4 & $(0,3)$ & \\
\hline Aucune comorbidité & 4560 & $(52,2)$ & 678 & $(49,5)$ & \multirow{3}{*}{0,0629} \\
\hline Comorbiditéd $^{d}$ & 4165 & $(47,6)$ & 690 & $(50,4)$ & \\
\hline Données manquantes & 19 & $(0,2)$ & 2 & $(0,2)$ & \\
\hline Aucun risque ${ }^{e}$ & 1087 & $(12,4)$ & 103 & $(7,5)$ & \multirow{4}{*}{$<0,0001$} \\
\hline Risque faible ${ }^{e}$ & 3566 & $(40,8)$ & 499 & $(36,4)$ & \\
\hline Risque modérée & 3571 & $(40,8)$ & 625 & $(45,6)$ & \\
\hline Risque élevée & 520 & $(6,0)$ & 143 & $(10,4)$ & \\
\hline \multicolumn{6}{|l|}{ Femmes } \\
\hline Totaux & 14708 & $(87,9)$ & 2020 & $(12,1)$ & \multirow{5}{*}{$<0,0001$} \\
\hline N’a jamais fumé & 12320 & $(83,8)$ & 1507 & $(74,6)$ & \\
\hline Fumeuse (quotidienne ou occasionnelle) & 2375 & $(16,2)$ & 512 & $(25,4)$ & \\
\hline Ancienne fumeuse & 5111 & $(34,8)$ & 947 & $(46,9)$ & \\
\hline Données manquantes & 13 & $(0,1)$ & 1 & $(0,1)$ & \\
\hline $\mathrm{IMC}<25 \mathrm{~kg} / \mathrm{m}^{2}$ & 5814 & $(39,5)$ & 973 & $(48,2)$ & \multirow{3}{*}{0,0035} \\
\hline $\mathrm{IMC} \geq 25 \mathrm{~kg} / \mathrm{m}^{2}$ & 8842 & $(60,1)$ & 1043 & $(51,6)$ & \\
\hline Données manquantes & 52 & $(0,4)$ & 4 & $(0,2)$ & \\
\hline Aucune comorbidité & 8551 & $(58,1)$ & 1242 & $(61,5)$ & \multirow{3}{*}{$<0,0001$} \\
\hline Comorbiditéd $^{d}$ & 6128 & $(41,7)$ & 772 & $(38,2)$ & \\
\hline Données manquantes & 29 & $(0,2)$ & 6 & $(0,3)$ & \\
\hline Aucun risque $\mathrm{e}^{\mathrm{e}}$ & 3540 & $(24,1)$ & 521 & $(25,8)$ & \multirow{4}{*}{$<0,0001$} \\
\hline Risque faible $e^{e}$ & 5673 & $(38,6)$ & 799 & $(39,6)$ & \\
\hline Risque modérée & 4813 & $(32,7)$ & 572 & $(28,3)$ & \\
\hline Risque élevée & 682 & $(4,6)$ & 128 & $(6,3)$ & \\
\hline
\end{tabular}

Abréviations : IMC, indice de masse corporelle; WCRF, World Cancer Research Fund.

Remarque : Les résultats sont présentés sous forme de pourcentage total pour chaque colonne.

${ }^{a}$ Les risques multiples ont été évalués à l'aide des critères suivants : consommation actuelle de tabac (occasionnelle ou quotidienne), taille corporelle (en surpoids ou obèse, soit un IMC $>25 \mathrm{~kg} / \mathrm{m}^{2}$ ) et comorbidité.

${ }^{b}$ Le respect des lignes directrices correspond à $\leq 2$ verres par jour chez les hommes et à $\leq 1$ verre par jour chez les femmes. Le dépassement des lignes directrices correspond à $>2$ verres par jour chez les hommes et $>1$ verre par jour chez les femmes.

' Le test de Khi carré a été utilisé pour les variables nominales, et l'analyse de variance à un facteur a été utilisée pour les variables continues.

d La comorbidité se définit comme l'autodéclaration de l'une des maladies chroniques suivantes : hypertension artérielle, angor, taux de cholestérol élevé, crise cardiaque, AVC, diabète, polypes du côlon, rectocolite hémorragique et cirrhose hépatique.

e Catégories de niveau de risque : aucun risque (les participants ne répondaient à aucun des critères ci-dessus, soit n'étaient pas fumeurs, avaient un IMC $<25 \mathrm{~kg} / \mathrm{m}^{2}$ et n'avaient déclaré aucune maladie chronique), risque faible (répondaient à un des trois critères ci-dessus), risque modéré (répondaient à deux des trois critères ci-dessus) et risque élevé (répondaient aux trois critères ci-dessus).

le World Cancer Research Fund/American Institute for Cancer Research (WCRF/ AICR). Toutefois, il est à noter que la cohorte incluait seulement des adultes de 35 ans et plus, ce qui exclut les 20 à 34 ans, dont on sait qu'ils sont les plus grands consommateurs d'alcool au Canada ${ }^{23}$. À l'échelle mondiale, la prévalence de la consommation d'alcool est en augmentation et demeure une préoccupation de santé publique $^{1}$. La consommation excessive d'alcool est largement reconnue comme étant nuisible à la santé1,5,6,8,9,22,47,48. Une méta-analyse récente a conclu qu'environ 34000 décès liés au cancer à travers le monde pourraient être attribués à une " faible " consommation d'alcool (soit moins de $12,5 \mathrm{~g}$ d'éthanol ou 1 verre ou moins par jour) en $2004^{49}$. Les effets nocifs de la consommation d'alcool sur la santé pourraient être sous-estimés par rapport à ceux découlant du tabagisme : on a estimé qu'en 2000 l'alcool avait entraîné une charge mondiale de morbidité semblable à celle du tabagisme ${ }^{8,48}$. Les résultats récents en recherche ne montrent pas que la consommation d'alcool aurait des effets protecteurs sur l'organisme ${ }^{18,50-52}$ et certaines études antérieures ayant étayé l'existence d'" effets protecteurs » de la consommation d'alcool étaient défaillantes sur le plan méthodologique $^{53-58}$ - en fait, ce sujet demeure controversés1,55,59-62.

Une grande proportion de participants à l'étude ont indiqué consommer de l'alcool en quantité faible ou modérée $(0,1$ à 29,9 g d'éthanol par jour ou de moins de 1 verre par jour à 2 verres par jour) et pourraient ne pas être conscients des effets néfastes potentiels de quantités d'alcool dans l'organisme certes faibles mais régulières. Des recherches plus approfondies concernant la relation entre la consommation d'alcool à faible risque et les effets sur la santé sont nécessaire pour caractériser de façon plus précise la limite entre les risques et les bienfaits de la consommation d'alcool au sein de divers groupes de population. Il est probable que les recommandations actuelles ne sont pas assez précises pour tenir compte des variations interindividuelles, de la prédisposition à certaines maladies et des seuils de tolérance.

Comme l'a déjà souligné l'Organisation panaméricaine de la santé et le WCRF, les comportements liés à la consommation d'alcool diffèrent considérablement selon le $\operatorname{sexe}^{3,47}$. Dans notre étude, les hommes consommaient de l'alcool plus fréquemment et en plus grande quantité que les femmes. Ils étaient aussi deux fois plus susceptibles que les femmes de faire état d'une consommation quotidienne d'alcool. Cette différence entre les sexes a été observée dans des études antérieures en population $^{3,47}$ et dans des études transnationales ${ }^{63,64}$, qui ont révélé une prévalence plus élevée de profils de consommation d'alcool nuisibles parmi les hommes, spécialement en ce qui a trait au volume total et aux habitudes de consommation à risque $^{63-65}$. Des études semblables ont 
également montré que la charge de morbidité attribuable à la consommation d'alcool (c'est-à-dire le cancer, la cirrhose hépatique, les troubles neuropsychiatriques, etc.) est 5 fois plus élevée chez les hommes que chez les femmes, avec un rapport de mortalité de 10 pour 1 en comparaison des femmes ${ }^{8}$. La plus grande consommation observée chez les hommes pourrait être attribuable à des facteurs biopsychosociaux $^{63}$. Dans le même ordre d'idées, nous avons constaté que davantage d'hommes consommaient des quantités élevées à la fois d'alcool et de tabac, amplifiant ainsi le risque d'effets néfastes sur leur santé et de maladie. Les hommes comme les femmes outrepassant les lignes directrices de consommation d'alcool avaient plus tendance à fumer et avaient, de manière générale, des profils plus à risque que ceux qui respectaient les recommandations en vigueur.

Les analyses exploratoires de cette recherche semblent indiquer que certaines maladies chroniques et comorbidités sont plus fréquentes chez les individus outrepassant les recommandations en matière de consommation d'alcool du WCRF/AICR, particulièrement chez les hommes. Par conséquent, les fournisseurs de soins de santé et les programmes publics devraient privilégier une optique de réduction des risques en déterminant quelles stratégies seraient les plus appropriées pour divers groupes d'individus. Il faudrait tout particulièrement intervenir auprès de populations identifiées comme ayant des habitudes de consommation d'alcool « à risque ». En raison des preuves accablantes qui démontrent la relation entre la quantité d'alcool consommée et le risque de développer une maladie chronique, dont le cancer, les messages de santé publique devraient non seulement viser plus spécifiquement les individus qui auraient déjà un profil à risque élevé, mais aussi continuer à mettre l'accent sur la prévention de la consommation excessive d'alcool et à encourager la consommation à faible risque pour les personnes qui choisissent de boire. Les prochaines analyses fondées sur l'Alberta's Tomorrow Project cibleront la relation entre les habitudes de consommation d'alcool à long terme et l'incidence du cancer et d'autres maladies chroniques au sein de cette cohorte.

\section{Limites}

Il est important de souligner que notre étude présente plusieurs limites. La cohorte de l'Alberta's Tomorrow Project n'inclut pas les jeunes adultes (moins de 35 ans), qui sont connus pour leur consommation d'alcool plus élevée en comparaison des adultes d'âge moyen ${ }^{31,34,66}$. Par conséquent, ces estimations reflètent seulement la population adulte de l'Alberta de 35 à 69 ans. Bien que l'étude Alberta's Tomorrow Project ait été conçue de manière à être géographiquement représentative de la population adulte de l'Alberta, aucune stratégie d'échantillonnage pondérée n'a été employée dans la conception de la cohorte. De plus, le recrutement initial effectué à l'aide de méthodes de composition aléatoire a produit un taux de réponse de $48,4 \%$ et il est impossible de savoir dans quelle mesure les répondants diffèrent des non-répondants, étant donné qu'aucune donnée n'a été recueillie auprès de ceux qui n'ont pas pris part à l'étude. Bien que nous pensions que nos résultats soient largement généralisables aux adultes de l'Alberta, ces données ne devraient pas être considérées comme représentatives de la population de l'Alberta. L'exclusion des Albertains de moins de 35 ans pourrait aussi expliquer la plus faible proportion de participants à l'étude Aberta's Tomorrow Project consommant de l'alcool en excès sur la base des recommandations du WCRF que ce que les autres données de surveillance nationale indiquent ${ }^{31,34,66}$. De plus, les résultats de nos analyses se fondent sur les réponses des participants à des questionnaires d'autodéclaration. Certaines questions délicates, comme celles en lien avec la consommation d'alcool, peuvent engendrer des erreurs de classification en raison de la sous-estimation et de la sous-déclaration de la consommation réelle ${ }^{3,8}$. Une analyse non publiée de l'Enquête sur les toxicomanies au Canada de 2004 a établi que les répondants indiquaient seulement le tiers, en moyenne, de ce qu'ils buvaient si on se réfère aux ventes d'alcool officielles $^{67}$. L'une des limites de l'utilisation du Diet History Questionnaire pour l'évaluation de la consommation d'alcool est qu'il ne cerne pas adéquatement les habitudes de consommation épisodique excessive d'alcool (« beuverie »), ce qui peut engendrer une sous-estimation de la consommation totale d'alcool. Néanmoins de nombreuses autres études bien conçues ont évalué la consommation d'alcool de cette manière, notamment la Nurses' Health Study ${ }^{41}$ et la Health Professionals Follow-up Study ${ }^{12}$, toutes deux étant d'importantes études de cohortes prospectives continues ${ }^{42}$.

\section{Conclusion}

Malgré de possibles sous-déclarations, $84 \%$ des participants à notre étude ont indiqué avoir consommé de l'alcool au cours de la dernière année. Les hommes avaient une consommation médiane (écart interquartile) de 5,9 $(14,8) \mathrm{g}$ par jour et les femmes, de 2,1 $(5,8)$ g par jour. Environ $14 \%$ des hommes et $12 \%$ des femmes outrepassaient les recommandations en matière de consommation d'alcool pour la prévention du cancer. De plus, de plus grands volumes de consommation d'alcool ont été associés au tabagisme et à des profils de comportements à risque élevé, aussi bien chez les hommes que chez les femmes (ensemble, $p<0,0001)$. Les messages de santé publique continuant à encourager une consommation minimale ou à faible risque sont essentiels pour la promotion de la modération parmi les personnes qui choisissent de consommer de l'alcool.

\section{Remerciements}

L'étude Alberta's Tomorrow Project est réalisable seulement grâce à l'engagement de ses participants, de son personnel et de ses bailleurs de fonds : l'Alberta Cancer Foundation, le Partenariat canadien contre le cancer, l'Alberta Cancer Prevention Legacy Fund (administré par Alberta Innovates - Health Solutions) et un important appui non financier d'Alberta Health Services. Cependant, les points de vue exprimés dans cette étude reflètent ceux des auteurs et non ceux de l'étude Alberta's Tomorrow Project ou de ses bailleurs de fonds. Les données issues de l'Enquête sur la santé dans les collectivités canadiennes sont fournies " telles quelles ", et Statistique Canada ne donne aucune garantie expresse ou implicite, que ce soit par exemple de la qualité d'utilisation ou de l'adéquation à une fin particulière. Statistique Canada ne peut en aucun cas être tenu responsable de dommages directs, particuliers, indirects, consécutifs ou autres. Christine Friedenreich détient une bourse de chercheure principale Health Senior Scholar d'Alberta Innovates - Health Solutions et est titulaire de la chaire de recherche sur le cancer du sein dans le cadre du Week-end pour vaincre les cancers féminins de l'Alberta Cancer Foundation. Darren Brenner détient une bourse de développement de carrière en prévention du cancer de la Société canadienne du cancer.

\section{Conflits d'intérêts}

Aucun conflit d'intérêts n'a été signalé. 


\section{Contributions des auteurs et avis}

DRB, PJR et CMF étaient responsables de l'élaboration de l'étude. CMF, DRB, PJR, $\mathrm{AEP}, \mathrm{TRH}$ et $\mathrm{AA}$ ont contribué de manière importante à la conception de l'étude et à l'interprétation des données. AA a effectué les analyses. DRB et TRH ont joué un rôle majeur dans la rédaction du manuscrit. Tous les auteurs ont lu et approuvé le manuscrit final et sont garants de son contenu.

Le contenu de cet article et les opinions qui y sont exprimées n'engagent que les auteurs et ne représentent pas forcément les opinions du Gouvernement du Canada.

\section{Références}

1. Organisation mondiale de la santé (OMS). Rapport de situation mondial sur l'alcool et la santé [2014]. Genève : OMS; 2014.

2. Danaei G, Vander Hoorn S, Lopez AD, Murray CJ, Ezzati M. Causes of cancer in the world: comparative risk assessment of nine behavioural and environmental risk factors. Lancet. 2005;366: 1784-1793.

3. World Cancer Research Fund/American Institute of Cancer Research (WCRF/ AICR). Food, nutrition, physical activity, and the prevention of cancer: a global perspective. World Cancer Res Fund Int 2007;517.

4. IARC Monographs on the Evaluation of Carcvinogenic Risks to Humans PREAMBLE. 2006.

5. Fedirko V, Tramacere I, Bagnardi V, et al. Alcohol drinking and colorectal cancer risk: an overall and doseresponse meta-analysis of published studies. Ann Oncol. 2011;22:1958-1972.

6. Islami F, Tramacere I, Rota $\mathrm{M}$, et al. Alcohol drinking and laryngeal cancer: overall and dose-risk relation asystematic review and meta-analysis. Oral Oncol. 2010;46:802-810.

7. Baan R, Straif K, Grosse Y, et al. Carcinogenicity of alcoholic beverages. Lancet Oncol. 2007;8:292-293.
8. Rehm J, Room R, Monteiro M, et al. Chapter 12: Alcohol use. In: Alcohol. 2004, pp 959-1108.

9. Corrao G, Bagnardi V, Zambon A, La Vecchia C. A meta-analysis of alcohol consumption and the risk of 15 diseases. Prev Med (Baltim). 2004;38: 613-619.

10. Grundy A, Poirier AE, Khandwala F, McFadden A, Friedenreich CM, Brenner DR. Cancer incidence attributable to alcohol consumption in Alberta, Canada in 2012. Can Med Assoc J Open. 2016;4:E507-514.

11. Rimm EB, Klatsky A, Grobbee D, Stampfer MJ. Review of moderate alcohol consumption and reduced risk of coronary heart disease: is the effect due to beer, wine, or spirits. BMJ. 1996;312:731-736.

12. Rimm EB, Giovannucci EL, Willett WC, et al. Prospective study of alcohol consumption and risk of coronary disease in men. Lancet. 1991;338:464-468.

13. Mukamal KJ, Jensen MK, Grønbæk M, et al. Drinking frequency, mediating biomarkers, and risk of myocardial infarction in women and men. Circulation. 2005;112:1406-1413.

14. Collins MA, Neafsey EJ, Mukamal KJ, et al. Alcohol in moderation, cardioprotection and neuroprotection: epidemiological considerations and mechanistic studies. Alcohol Clin Exp Res. 2009;33:206-219.

15. Beulens JWJ, Van der Schouw YT, Bergmann MM, et al. Alcohol consumption and risk of type 2 diabetes in European men and women: Influence of beverage type and body size. The EPIC-InterAct study. J Intern Med. 2012;272:358-370.

16. Metcalf PA, Scragg RKR, Jackson R. Light to moderate alcohol consumption is protective for type 2 diabetes mellitus in normal weight and overweight individuals but not the obese. J Obes. 2014; 2014. doi: 10.1155/2014 /634587.

17. Arranz S, Chiva-Blanch G, ValderasMartínez P, Medina-Remón A, Lamuela-Raventós RM, Estruch R. Wine, beer, alcohol and polyphenols on cardiovascular disease and cancer. Nutrients. 2012;4:759-781.
18. Connor J. Alcohol consumption as a cause of cancer. Addiction. 2016;103: 153-160.

19. Rehm J, Shield K. Alcohol consumption. In: Stewart BW, Wild CB E (ed). World Cancer Report 2014. Lyon (France) : International Agency for Research on Cancer; 2014.

20. Klatsky AL, Udaltsova N, Li Y, Baer D, Nicole Tran H, Friedman GD. Moderate alcohol intake and cancer: the role of underreporting. Cancer Causes Control. 2014;25:693-699.

21. Toma A, Paré G, Leong DP. Alcohol and Cardiovascular Disease: How Much is Too Much? Curr Atheroscler Rep. 2017;19:13.

22. Bagnardi V, Rota M, Botteri E, et al. Alcohol consumption and site-specific cancer risk : a comprehensive dose response. Br J Cancer. 2014;112:580-593.

23. Agence de la santé publique du Canada (ACSP). Rapport de l'administrateur en chef de la santé publique sur l'état de la santé publique au Canada, 2015 : la consommation d'alcool au Canada. Ottawa (Ont.) : ASPC; 2016. doi: $10.1177 / 1469605310365110$.

24. Butt P, Beirness D, Gliksman L, Paradis C, Stockwell T. L'alcool et la santé au Canada : résumé des données probantes et directives de consommation à faible risque. Ottawa (Ont.) : Centre canadien de lutte contre l'alcoolisme et les toxicomanies, 2011 En ligne à : http://educalcool.qc.ca /wp-content/uploads/2013/05/NCAFR -Document-scientifique.pdf

25. The Canadian Centre on Substance Abuse. Canada's Low Risk Alcohol Drinking Guidelines. Alcohol Drink. Guidel. 2013;4-5.

26. Santé Canada. Enquête sur les toxicomanies au Canada (ETC) : une enquête nationale sur la consommation d'alcool et d'autres drogues par les Canadiens : la prévalence de l'usage et les méfaits. [Internet]. Ottawa (Ont.) : Santé Canada; 2004 [consultation le 4 janvier 2017]. En ligne à : http://www.ccsa.ca/Resource \%20Library/ccsa-004029-2005.pdf 
27. Santé Canada. Prévalence de la consommation d'alcool et le dépassement des Directives de consommation d'alcool à faible risque, selon l'âge, ETC de 2004 et ESCCAD de 2008-2012. Enquête de surveillance canadienne de la consommation d'alcool et de drogues [Internet]. Ottawa (Ont.) : Statistique Canada; 2014 [consultation le 4 janvier 2017]. En ligne à : https:// www.canada.ca/fr/sante-canada /services/preoccupations-liees-sante /prevention-traitement-toxicomanie /statistiques-consommation-drogues -alcool/enquete-surveillance-canadienne - consommation-alcool-drogues -sommaire-resultats-tableaux-2012 .html

28. Statistique Canada. Les indicateurs de l'alcool, selon le sexe et l'âge, 2013. Tableaux supplémentaires, l'ECTAD, Annuel 2013 (février à décembre, 2013) [Internet]. Ottawa (Ont.) : Statistique Canada; 2013 [consultation le 4 janvier 2017]. En ligne à : https:// www.canada.ca/fr/sante-canada /services/enquete-canadienne-tabac -alcool-et-drogues/2013-tableaux -supplementaires.html

29. Statistique Canada. Enquête sur la santé dans les collectivités canadiennes : Information détaillée pour 2012. [Internet]. Ottawa (Ont.) : Statistique Canada; 2012 [consultation le 4 janvier 2017]. En ligne à : http:// www23.statcan.gc.ca/imdb/p2SV_f .pl? Function $=$ getSurvey\&SDDS $=5015$ \&Item_Id = 119790

30. Statistique Canada. Enquête sur la santé dans les collectivités canadiennes (ESCC) : Information détaillée pour 2005 (cycle 3.1) [Internet]. Ottawa (Ont.) : Statistique Canada; 2006 [consultation le 4 janvier 2017]. En ligne à : http://www23.statcan .gc.ca/imdb/p2SV_f.pl?Function = getSurvey\&Id $=2264231$

31. Santé Canada. Enquête de surveillance canadienne de la consommation d'alcool et de drogues (ESCCAD), 2008. Ottawa (Ont.) : Santé Canada; 2008 [consultation le 4 janvier 2017]. En ligne à http://www.hc-sc.gc.ca /hc-ps/drugs-drogues/stat/_2008 /summary-sommaire-fra.php
32. Santé Canada. Enquête de surveillance canadienne de la consommation d'alcool et de drogues (ESCCAD), 2009. Ottawa (Ont.) : Santé Canada; 2009 [consultation le 4 janvier 2017]. En ligne à http://www.hc-sc.gc.ca/hc-ps /drugs-drogues/stat/_2009/summary -sommaire-fra.php

33. Santé Canada. Enquête de surveillance canadienne de la consommation d'alcool et de drogues (ESCCAD), 2010. Ottawa (Ont.) : Santé Canada; 2011 [consultation le 4 janvier 2017]. En ligne à : http://www.hc-sc.gc.ca/hc-ps /drugs-drogues/stat/_2010/summary -sommaire-fra.php

34. Santé Canada. Enquête de surveillance canadienne de la consommation d'alcool et de drogues (ESCCAD), 2011 [Internet]. Ottawa (Ont.) : Santé Canada; 2011 [consultation le 4 janvier 2017]. En ligne à http://www.hc-sc .gc.ca/hc-ps/drugs-drogues/stat/_2011 /summary-sommaire- fra.php

35. Bryant H, Robson PJ, Ullman R, Friedenreich C, Dawe U. Populationbased cohort development in Alberta, Canada: a feasibility study. Chronic Dis Can. 2006; 27: 51-59.

36. Robson PJ, Solbak N, Haig T. Cohort profile: design, methods, and demographics from phase I of Alberta's Tomorrow Project cohort. Can Med Assoc J Open 4. 2016; E515-E527.

37. Csizmadi I, Kahle L, Ullman R, et al. Adaptation and evaluation of the National Cancer Institute's Diet History Questionnaire and nutrient database for Canadian populations. Public Health Nutr. 2007;10:88-96.

38. Friedenreich CM, Courneya KS, Bryant HE. The lifetime total physical activity questionnaire: development and reliability. Med Sci Sports Exerc. 1998;30: 266-274.

39. Friedenreich CM, Courneya KS, Neilson HK, et al. Reliability and validity of the Past Year Total Physical Activity Questionnaire. Am J Epidemiol. 2006;163:959-970.

40. National Institutes of Health. Diet History Questionnaire. 2007.
41. Mostofsky E, Mukamal KJ, Giovannucci EL, Stampfer MJ, Rimm EB. Key Findings on alcohol consumption and a variety of health outcomes from the Nurses' Health Study. Am J Public Health. 2016;106:1586-1591.

42. Cao Y, Willett WC, Rimm EB, Stampfer MJ, Giovannucci EL. Light to moderate intake of alcohol, drinking patterns, and risk of cancer: results from two prospective US cohort studies. BMJ. 2015;351: h4238.

43. Centre de toxicomanie et de santé mentale. L'alcool. [Internet]. Toronto (Ont.) : CAMH; 2012. En ligne à : http://www.camh.ca/fr/hospital /health_information/a_z_mental_ health_and_addiction_information /alcohol/Pages/alcohol.aspx.

44. World Cancer Research Fund/American Institute for Cancer Research. Food, nutrition, physical activity, and the prevention of cancer: a global perspective. Washington DC : American Institute for Cancer Research; 2007. doi: 978-0-9722522-2-5.

45. Alberta Health Services. Alcohol and Health: Alcohol and Alberta. Edmonton, Alberta, 2015.

46. Canadian Centre on Substance Abuse. Alcohol. 2014.

47. Pan American Health Organization. Alcohol, gender, culture and harms in the Americas: PAHO Multicentric Study final report. [Internet]. Washington, DC, 2007. En ligne à : http://www .who.int/substance_abuse/publications /alcohol_multicentric_americas.pdf.

48. Rehm J, Mathers C, Popova S, Thavorncharoensap M, Teerawattananon Y, Patra J. Global burden of disease and injury and economic cost attributable to alcohol use and alcohol-use disorders. Lancet. 2009; 373:2223-2233.

49. Bagnardi V, Rota M, Botteri E, et al. Light alcohol drinking and cancer: A meta-analysis. Ann Oncol. 2013;24: 301-308.

50. Chikritzhs T, Fillmore K, Stockwell T. A healthy dose of scepticism: four good reasons to think again about protective effects of alcohol on coronary heart disease. Drug Alcohol Rev. 2009;28:441-444. 
51. Chikritzhs T, Stockwell T, Naimi T, Andreasson S, Dangardt F, Liang W. Has the leaning tower of presumed health benefits from 'moderate' alcohol use finally collapsed? (editorial). Addiction. 2015; 110:726-727.

52. Stockwell T, Naimi T. Study raises new doubts regarding the hypothesised health benefits of 'moderate' alcohol use. Evid Based Med. 2016; 21:156.

53. Fekjaer HO. Alcohol-a universal preventive agent? A critical analysis. Addiction. 2013;108:2051-57.

54. Naimi TS, Stockwell T, Zhao J, et al. Selection biases in observational studies affect associations between 'moderate' alcohol consumption and mortality. Addiction. 2016. doi: 10.1111/add.13451.

55. Jackson R, Broad J, Connor J, Wells $\mathrm{S}$. Alcohol and ischaemic heart disease: probably no free lunch. Lancet. 2005;366:1911-1912.

56. Naimi TS, Brown DW, Brewer RD, et al. Cardiovascular risk factors and confounders among nondrinking and moderate-drinking U.S. adults. Am J Prev Med. 2005;28:369-373.

57. Goldberg IJ. To drink or not to drink? N Engl J Med. 2003;348:163-164.

58. Naimi TS, Xuan Z, Brown DW, Saitz $R$. Confounding and studies of 'moderate' alcohol consumption: the case of drinking frequency and implications for low-risk drinking guidelines. Addiction. 2013;108:1534-1543.

59. Roerecke M, Rehm J. Alcohol consumption, drinking patterns, and ischemic heart disease: a narrative review of meta-analyses and a systematic review and meta-analysis of the impact of heavy drinking occasions on risk for moderate drinkers. BMC Med. 2014;12:182.

60. Stockwell T. A reply to Roerecke \& Rehm: Continuing questions about alcohol and health benefits. Addiction. 2013;108:428-429.

61. Stockwell T, Chikritzhs T. Commentary: Another serious challenge to the hypothesis that moderate drinking is good for health? Int $\mathrm{J}$ Epidemiol. 2013;42:1792-1794.
62. Bergmann MM, Rehm J, KlipsteinGrobusch K, et al. The association of pattern of lifetime alcohol use and cause of death in the European Prospective Investigation into Cancer and Nutrition (EPIC) study. Int J Epidemiol. 2013;42:1772-1790.

63. Wilsnack RW, Vogeltanz ND, Wilsnack SC, et al. Gender differences in alcohol consumption and adverse drinking consequences: cross-cultural patterns. Addiction. 2000;95:251-265.

64. Wilsnack RW, Wilsnack SC, Kristjanson AF, Vogeltanz-Holm ND, Gmel G. Gender and alcohol consumption: patterns from the multinational GENACIS project. Addiction. 2009; 104:1487-1500.

65. Hughes TL, Wilsnack SC, Kantor LW. The Influence of Gender and Sexual Orientation on Alcohol Use and Alcohol-Related Problems: Toward a Global Perspective. Alcohol Res. 2016; 38:121-132.

66. Sawka E, Huebert K, Malcolm C, Phare S, Adlaf E. Canadian Addiction Survey 2004: provincial differencesalcohol. 2007.

67. Stockwell T, Zhao J, Thomas G. Should alcohol policies aim to reduce total alcohol consumption? New analyses of Canadian drinking patterns. Addict Res Theory. 2009;17:135-151. 